\title{
Supersymmetry breaking and gauge mediation in models with a generic superpotential
}

\author{
Ryuichiro Kitano ${ }^{\dagger}$ and Yutaka Ookouchi* \\ $\dagger$ Theoretical Division T-2, Los Alamos National Laboratory, NM 87545, USA \\ * Perimeter Institute for Theoretical Physics, ON N2L2Y5, Canada
}

We present a general scheme for finding or creating a metastable vacuum in supersymmetric theories. By using the formalism, we show that there is a parameter region where a metastable vacuum exists in the Wess-Zumino model coupled to messenger fields. This model serves as a perturbative renormalizable model of direct gauge mediation. 


\section{Introduction}

If the theory to describe our universe possesses supersymmetry, the vacuum of the theory should be somewhat unusual. Supersymmetry, which is a part of the space-time symmetry, must be spontaneously broken in the vacuum whereas the rest of the spacetime symmetries, the Poincare symmetry, are kept unbroken. There have been many attempts to realize this hypothesis in four-dimensional field theories. For example, the ideas of dynamical supersymmetry breaking [1-3] and gauge mediation [4-9] (see also $[10,11]$ for review and relevant references) have attracted much attention because of their beautiful concepts. However, a rather contrived set-up was necessary because generic supersymmetric theories have a stable supersymmetric vacuum.

The recent discovery of metastable vacua in SQCD [12] opened up new avenue for a realistic model building because of its simplicity. The idea of living in a metastable vacuum allows us to consider vector-like models (for earlier works see [13]) whose nonperturbative effects have been well understood [14]. String-theory dualities can also provide useful tools to analyze such theories and their vacuum structures. Various works on this subject have been done (see e.g. [15]), including models with extended supersymmetry [16-18] and geometrical realization of metastable vacua in string theories [19-26]. Also, the idea of metastable vacua drastically simplified a way of gauge mediation and simple successful models have been proposed [27-39]. Motivated by the fact that the low energy theories of many supersymmetry breaking models are identical to the O'Raifeartaigh model, the vacuum of generalized O'Raifeartaigh models have been studied in [40-45].

The usual steps to build a gauge-mediation model are as follows. We first construct/prepare a model of supersymmetry breaking. We introduce messenger fields which carry quantum numbers of the standard-model gauge group, and let them couple to the supersymmetry breaking model. (Or, if possible we identify messenger fields in the supersymmetry breaking model.) At this stage, we often encounter one of the following two problems: the appearance of a new unstable direction towards a new supersymmetric vacuum where the messenger fields acquire vacuum expectation values, or vanishing gaugino masses. Therefore, in either case, the final step is to deform the model so that the messenger fields are stabilized and the gauginos obtain masses. In short, we break supersymmetry, make it restored and try to break again, or we break supersymmetry and deform the model while avoiding the supersymmetry 
breaking vacuum to be destroyed. The first and the final steps are quite simplified when we allow the supersymmetry breaking vacuum to be metastable. However, as one can notice from this discussion, it may be a detour to start from a supersymmetry breaking model once we allow the metastability. There can be a possibility that one starts from a generic theory including the messenger fields and finds a (meta)stable minimum of the potential.

In this note, we present a transparent scheme for finding or creating a (meta)stable vacuum in general supersymmetric models. We derive general conditions for having a supersymmetry breaking vacuum by connecting different models by a coordinate transformation, which is an application of the method used in [16]. In particular, we find that there can be a metastable supersymmetry breaking vacuum in models with the canonical Kähler potential and a generic superpotential. For example, the Wess-Zumino model coupled to the messenger fields possesses a metastable vacuum if coefficients of the superpotential terms satisfy certain inequalities.

\section{Local equivalence to the Polonyi model}

In this section we develop a general method of creating a metastable supersymmetry breaking vacuum. Let us start with the following Lagrangian defined by

$$
\mathcal{L}=[K(z, \bar{z})]_{D}-\left([\epsilon z]_{F}+\text { h.c. }\right),
$$

where $z$ is a chiral superfield and $\epsilon$ is a parameter. This model (the Polonyi model [46]) has a stable SUSY breaking vacuum at $z=0$ if

$$
\begin{gathered}
\left.K_{z \bar{z}}\right|_{z=0}>0, \\
\left.K_{z z \bar{z}}\right|_{z=0}=\left.K_{z \bar{z} z}\right|_{z=0}=0, \\
\left.K_{z z \bar{z} \bar{z}}\right|_{z=0}<0, \\
\left.K_{z z \bar{z} \bar{z}}^{2}\right|_{z=0}-\left.\left|K_{z z z \bar{z}}\right|_{z=0}\right|^{2}>0 .
\end{gathered}
$$

We implicitly assumed that the function $K$ is differentiable at least four times at $z=0$. The condition in (2.1) is a sign convention of the kinetic term. As long as the sign is 
correct, we can normalize the $z$ field so that $\left.K_{z \bar{z}}\right|_{z=0}=1$. Also, (2.2) can always be satisfied by an appropriate shift of $z$. With this convention, the conditions (2.3) and (2.4) ensure the stability of the potential. For convenience, we define

$$
\left.\alpha \equiv K_{z z \bar{z} \bar{z}}\right|_{z=0},\left.\quad \beta \equiv K_{z z z \bar{z}}\right|_{z=0},
$$

with the canonical normalization of the field, $\left.K_{z \bar{z}}\right|_{z=0}=1$. The $\alpha$ parameter is a real number whereas $\beta$ is a complex number. The masses of the scalar components, $m_{R}$ and $m_{I}$, satisfy

$$
m_{R}^{2}+m_{I}^{2}=-2|\epsilon|^{2} \alpha, \quad m_{R}^{2} m_{I}^{2}=|\epsilon|^{4}\left(\alpha^{2}-|\beta|^{2}\right) .
$$

The vacuum energy at $z=0$ is

$$
\left.V\right|_{z=0}=|\epsilon|^{2}
$$

Now, we perform a field redefinition (or a coordinate transformation), $z \rightarrow X=$ $w^{-1}(z)$, where $w^{-1}(z)$ is the inverse of a holomorphic function $w(X)$. The function $w(X)$ is required to be analytic in the neighbor of $X=x_{0}$ where $w\left(x_{0}\right)=0$, and also the first derivative should not vanish $\left(\left.w_{X}\right|_{x_{0}} \neq 0\right)$ in order for the inverse function to be at least locally defined around the stable point $z=0$. Since we are interested only in the local structure of the potential, we do not need the transformation to be globally defined. The superpotential in terms of $X$ is given by

$$
W=\epsilon w(X)
$$

This transformation generates various kinds of (metastable) SUSY breaking model by simply choosing an arbitrary holomorphic function $w(X)$. Here, for simplicity, we restrict ourselves to the case that $w(X)$ is a function of a single chiral superfield, $X$. In a more general case, the discussion can be reduced to the single-field case by integrating out other degrees of freedom. In appendix we present a general discussion.

The stable point at $z=0$ maps to $X=x_{0}$. By assumption, we can expand the function $w(X)$ around $x_{0}$ :

$$
w(X)=\xi\left(x_{0}\right)\left(\Delta X+\frac{\xi_{2}\left(x_{0}\right)}{2} \Delta X^{2}+\frac{\xi_{3}\left(x_{0}\right)}{6} \Delta X^{3}+\cdots\right),
$$

where $\xi\left(x_{0}\right)=\left.w_{X}\right|_{x_{0}} \neq 0, \xi_{2}\left(x_{0}\right)=\left.w_{X X}\right|_{x_{0}} / \xi\left(x_{0}\right)$ and $\xi_{3}\left(x_{0}\right)=\left.w_{X X X}\right|_{x_{0}} / \xi\left(x_{0}\right)$, and $\Delta X=X-x_{0}$. We can take $\xi\left(x_{0}\right)=1$ which corresponds to the change of the 
function: $w(X) \rightarrow w(X) / \xi\left(x_{0}\right)$. The higher order terms of $O\left(\Delta X^{4}\right)$ are irrelevant for the discussion of the local stability. The expansion of the Kähler potential in terms of the $X$ field around $X=x_{0}$ has the following coefficients:

$$
\begin{gathered}
\left.K_{X X \bar{X}}\right|_{x_{0}}=\xi_{2}\left(x_{0}\right), \\
K_{X X \bar{X} \bar{X} \mid x_{0}}=\left|\xi_{2}\left(x_{0}\right)\right|^{2}+\alpha, \\
\left.K_{X X X \bar{X} \mid}\right|_{x_{0}}=\xi_{3}\left(x_{0}\right)+\beta .
\end{gathered}
$$

Here we take the canonical normalization of the $X$ field, $\left.K_{X \bar{X}}\right|_{x_{0}}=1$. Higher order terms are again irrelevant for the discussion. The meaning of the equations is the following. With a given superpotential and a Kähler potential for the $X$ field, there is a (meta)stable minimum at $X=x_{0}$ if it is possible to find $\alpha, \beta$, and $x_{0}$ which satisfy the above equations and the conditions in (2.3) and (2.4), i.e.,

$$
-\alpha>|\beta| .
$$

For $\beta=0$, the field redefinition $X \rightarrow w(X)$ defined by the coefficients, $\xi_{2,3}$, from (2.7) and (2.9) corresponds to the transformation to the Kähler normal coordinate (A.1) in our normalization.

Since there are five real equations for five real variables $\left(\alpha, \operatorname{Re}[\beta], \operatorname{Im}[\beta], \operatorname{Re}\left[x_{0}\right]\right.$, $\left.\operatorname{Im}\left[x_{0}\right]\right)$, there is generically a solution. These equations are particularly useful when we know the Kähler potential. In such a case, one can choose a point $x_{0}$, set $\beta=0$, and then fix $\xi_{2}, \xi_{3}$ and $\alpha$ by using the above equations. If $\alpha$ is negative, we can obtain a superpotential which creates a metastable vacuum at $X=x_{0}$. Note that this is not a fine-tuning of the parameters. Around the constructed solution, there is a region of the parameter space where a (meta)stable vacuum exists.

The coordinate transformation $z \rightarrow w^{-1}(z)$ can modify the global structure of the potential although the local stability is ensured in this formulation. For example, the Wess-Zumino model obtained by this transformation has SUSY vacua whereas the original Polonyi model does not. Conversely, if the transformation is globally defined, there is no SUSY vacuum because two models are the same. In the metastable case, the lifetime of the vacuum can be arbitrarily long by taking $\epsilon$ to be small.

\footnotetext{
*Or, one can fix Kähler terms if the superpotential is known.
} 
In general, this formulation can be used to find a metastable vacuum in a given model by checking if the model is locally equivalent to the Polonyi model, i.e., if a solution is constructable. By following the derivation inversely, one can see that the above condition is a necessary and sufficient condition for supersymmetry breaking. A necessary condition for having a metastable vacuum is therefore an existence of a point $x_{0}$ where

$$
\left.K_{X X \bar{X} \bar{X}}\right|_{x_{0}}<\left.\left.\left|K_{X X \bar{X}}\right|\right|_{x_{0}}\right|^{2} .
$$

An interesting observation is that there is a useful sufficient condition for the metastability. If the Kähler potential satisfies

$$
K_{X X \bar{X} \bar{X}}=0
$$

everywhere (except singular points) in the $X$ space, one can create a metastable vacuum anywhere by adding a suitable superpotential. $N=2$ supersymmetric theories are such examples [16].

\section{Gauge Mediation Models}

\subsection{General argument}

In this section we will construct models of direct gauge mediation where the particle content is supersymmetry breaking fields, $X_{i}$, and the messenger fields, $f_{k}, \tilde{f}_{k}$. We take the canonical Kähler potential:

$$
K=X^{i} X_{i}^{\dagger}+f^{k} f_{k}^{\dagger}+\tilde{f}^{k} \tilde{f}_{k}^{\dagger} .
$$

Suppose the superpotential includes interaction terms,

$$
\mathcal{W}=M(X)^{k l} f_{k} \tilde{f}_{l}+\epsilon W(X),
$$

where $M(X)$ and $W(X)$ are arbitrary holomorphic functions of $X_{i}$. For successful gauge mediation, the messengers should acquire masses at the vacuum. Thus we are interested in a parameter region where all the eigenvalues of the mass matrix $M(X)$ are non-vanishing and much larger than that of the field $X_{i}$ and $\sqrt{F_{X^{i}}}$. To achieve this, we 
take $\epsilon$ to be small, $\epsilon \ll 1$. At the low energy, the massive messengers can be integrated out and correction terms to the Kähler potential of the fields $X_{i}$ are generated:

$$
K=K_{c l}+\eta K_{1 l o o p}+\eta^{2} K_{2 l o o p}+\cdots
$$

where $\eta$ is one loop factor. They yield non-zero curvature in the $X$ space, which allows us to use the method shown in the previous section.

Suppose that there is a point $x_{0}$ where the sectional curvature is postive definite, (for a model with single $X,\left.K_{X \bar{X} X \bar{X}}\right|_{x_{0}}<\left.\left|K_{X X \bar{X}}\right|_{x_{0}}\right|^{2}$ ). Then as we have seen, by taking the superpotential $W(X)$ appropriately, we can create a metastable vacuum at the point $x_{0}$. At the supersymmetry breaking minimum,

$$
\left\langle X^{i}\right\rangle=x_{0}^{i}+\theta^{2} F_{X^{i}}
$$

the messenger masses are easily read off from the original Lagrangian,

$$
\mathcal{W}=\left[M\left(x_{0}\right)+\theta^{2} F_{X_{i}} \partial_{i} M\left(x_{0}\right)\right]^{k l} f_{k} \tilde{f}_{l}+\cdots
$$

To study the idea in more detail, let us focus on a simple example.

\subsection{Simplest example}

We consider the case with a single $X$ field and a pair of $f$ and $\tilde{f}$. Take $M(X)$ be linear and $W(X)$ be a cubic function of $X$. The messenger is $\mathbf{5}+\overline{\mathbf{5}}$ in $S U(5)$.

$$
\mathcal{W}=\lambda X f \tilde{f}+W_{\text {cubic }}(X)
$$

This is the general renormalizable theory with the singlet field $X$ and the messenger fields $f$ and $\tilde{f}$. The bare mass term $m f \tilde{f}$ can be eliminated by a shift of the $X$ field. After integrating out the messenger fields, the Kähler potential of the $X$ is modified and the metric of it can be written as

$$
g \simeq e^{\log Z_{X}(X)}
$$

As in [54] by expanding the wavefunction renormalization in small expansion paramters $\eta \ll \eta \log \frac{|X|}{\Lambda} \ll 1$, the effective Kähler metric is given by

$$
g(X)=g_{0}(\mu)\left(1+\left(\eta A^{(1)}+\eta^{2} B^{(1)}\right) \log \frac{|X|^{2}}{\mu^{2}}+\eta^{2} A^{(2)}\left(\log \frac{|X|^{2}}{\mu^{2}}\right)^{2}+\cdots\right)
$$


where the cut-off $\Lambda$ is renormalized by $g_{0}$. In this specific model, the one-loop factor $\eta$ defined above is $N_{m}|\lambda|^{2} / 16 \pi^{2}$ where $N_{m}$ is number of messengers, which is 5 in our present model. To controll our metastable state, we have to keep two-loop correction terms. For computation of these correction terms, we take an advantage of thinking large $X$ region [52-54]. Since we are interested in a parameter region where mass of $X$ is very small compared to the one of the messengers, which guarantee that vev of $X$ is much larger than SUSY breaking scale, $\langle X\rangle^{2} \gg F$. In this case, we need only to know dominant log contributions. The other terms are suppressed by $F /\langle X\rangle^{2}$. These $\log$ contributions can be computed by essentially following the paper $[50,51,54]$. The $A^{(1)}$ and $A^{(2)}$ are computed respectively by discontinuity of the one-loop anomalous dimension and discontinuity of the derivative of it with respect to the $\mathrm{RG}$ time, $t=\log \mu$ at the threshold scale $\mu=x_{0}$. On the other hand, the coefficient $B^{(1)}$ is given by a discontinuity of the two-loop anomalous dimension. Using the known formulae for one-loop and two-loop anomalous dimension (see for example [55]),

$$
\gamma_{X}^{\text {one }}=\frac{N_{m}|\lambda|^{2}}{16 \pi^{2}}, \quad \gamma_{X}^{\text {two }}=-\frac{N_{m}|\lambda|^{4}}{\left(16 \pi^{2}\right)^{2}},
$$

where $\gamma_{X} \equiv-\frac{1}{2} \log Z_{X}$, we can compute the coefficients in the Kähler metric (3.3) explicitly,

$$
\begin{aligned}
\eta A^{(1)} & =\Delta \gamma_{X}^{\text {one }}=-\eta \\
\eta^{2} B^{(1)} & =\Delta \gamma_{X}^{\mathrm{two}}=\eta^{2} \frac{1}{N_{m}} \\
\eta^{2} A^{(2)} & =\frac{1}{2}\left(\Delta \gamma_{X}^{\text {one }}\right)^{2}+\frac{1}{4} \Delta\left(\frac{\partial \gamma_{X}^{\text {one }}}{\partial t}\right)=-\eta^{2} \frac{1}{N_{m}}
\end{aligned}
$$

where we define $\Delta O \equiv O\left(t_{x_{0}}^{(-)}\right)-O\left(t_{x_{0}}^{(+)}\right)$. From this, we see that the sign of $A^{(2)}$ is negative, thus the condition $\left.K_{X \bar{X} X \bar{X}}\right|_{x_{0}}<\left.\left|K_{X X \bar{X}}\right|_{x_{0}}\right|^{2}$ is hold everywhere in the field space. Following the method shown in section 2, we can create a metastable supersymmetry breaking vacuum anywhere we want in field space. The expansion coefficients around $X=x_{0}$ are

$$
\begin{gathered}
K_{\left.X X \bar{X}\right|_{x_{0}}}=\frac{\eta}{x_{0}} A^{(1)}+\mathcal{O}\left(\eta^{2}\right), \\
K_{\left.X X \bar{X} \bar{X}\right|_{x_{0}}}=\frac{2 \eta^{2}}{\left|x_{0}\right|^{2}} A^{(2)}+\mathcal{O}\left(\eta^{3}\right), \\
K_{\left.X X X \bar{X}\right|_{x_{0}}}=-\frac{\eta}{x_{0}^{2}} A^{(1)}+\mathcal{O}\left(\eta^{2}\right),
\end{gathered}
$$


where the $X$ field is canonically (re)normalized, $\left.K_{X \bar{X}}\right|_{x_{0}}=1$. A metastable vacuum at $X=x_{0}$ can be created by adding a superpotential:

$$
W_{\text {cubic }}=c_{1}\left(X-x_{0}\right)+\frac{c_{2}}{2}\left(X-x_{0}\right)^{2}+\frac{c_{3}}{6}\left(X-x_{0}\right)^{3},
$$

with

$$
\begin{aligned}
& \frac{c_{2}}{c_{1}}=\left.K_{X X \bar{X}}\right|_{x_{0}} \\
& \left.\left.\left|\frac{c_{3}}{c_{1}}-K_{X X X \bar{X}}\right|_{x_{0}}|<| K_{X X \bar{X}}\right|_{x_{0}}\right|^{2}-\left.K_{X \bar{X} X \bar{X}}\right|_{x_{0}} .
\end{aligned}
$$

The value of $c_{1}$ can be arbitrary.

We can discuss the parameter region in terms of a set of parameters whose mass dimensions are more transparent:

$$
W_{\text {cubic }}=m^{2}\left(X+\frac{X^{2}}{2 M}+\frac{\kappa X^{3}}{6 M^{2}}\right) .
$$

The values of the dimensionful parameters $m$ and $M$ are arbitrary as long as $m \ll M$. For $\eta \ll 1$, the parameter $\kappa$ needs to be $O\left(\eta^{-1}\right)$ and the VEV $x_{0}$ is of $O(\eta)$ in the unit of $M$. The condition in (3.7) tells us that the $\kappa$ parameter should be within a narrow range: $\kappa=1 /(4 \eta)+3 / 8+1 / 4 N_{m} \pm O(\eta)$. One needs a rather large $\eta$ to avoid the fine-tuning.

For a not very small $\eta$, i.e., for a not very large $\kappa$, the hierarchical structure of the parameters in (3.8) can naturally be obtained, for example, from a theory with a small breaking of $R$-symmetry. If we assign an $R$-charge $x$ to the $X$ field and introduce a spurion for the $R$-breaking $\phi_{R}$ with the same charge $x$, then the $R$-invariant superpotential has the following structure:

$$
W_{\text {cubic }} \sim\left(\frac{\phi_{R}}{\Lambda}\right)^{(2-3 x) / x}\left(\phi_{R}^{2} X+\phi_{R} X^{2}+X^{3}\right),
$$

with $O(1)$ coefficients. f The scale $\Lambda$ is a cut-off of the theory. For $\phi_{R} \ll \Lambda$ and $x<2 / 3$, the above superpotential has the desired structure where $M \sim \phi_{R}$ and $m^{2} \sim M^{2}\left(\phi_{R} / \Lambda\right)^{(2-3 x) / x}$.

\footnotetext{
${ }^{\dagger}$ A naive estimation from (3.7) gives $\kappa=O(1 / \eta) \pm O(1)$. However, there is a cancellation between coefficients in the calculation from (3.5) to (3.8). If we include an $X^{4}$ term, the $\kappa$ parameter can be arbitrary. However, the same degree of fine-tuning is necessary between $\kappa$ and the coefficient of the $X^{4}$ term.

${ }^{\ddagger}$ We assumed that there is no terms with inverse powers of $\phi_{R}$.
} 


\section{Acknowledgements}

We would like to thank P. Argyres, F. Cachazo, H. Ooguri, K. Maruyoshi, J. Marsano for discussions. Research at Perimeter Institute for Theoretical Physics is supported in part by the Government of Canada through NSERC and by the Province of Ontario through MRI. YO would like to thank the University of Cincinnati and University of Pisa for kind hospitality.

\section{A Model with multiple chiral superfields $X_{i}$}

In this section, we generalize the argument shown in section 2 to models with multiple chiral superfields $X_{i}$ applying the method used in [16]. Suppose the Kähler potential is a generic function of chiral superfields $K\left(X_{i}\right)$ and pick a point $X_{0}^{i}$ in the $X$ space. In the Kähler normal coordinate [47,48],

$$
\omega^{i}=\Delta X^{i}+\left.\frac{1}{2} \Gamma_{j k}^{i}\right|^{j} \Delta X^{k}+\frac{1}{6} g^{m \bar{l}} \partial \Gamma_{\bar{l} i j} \mid \Delta X^{i} \Delta X^{j} \Delta X^{k}+\cdots,
$$

where $\Delta=X-X_{0}$, the inverse of the metric is

$$
g^{i \bar{j}}=g^{i \bar{j}}\left|+R_{k \bar{l}}^{i \bar{j}}\right| \omega^{k} \bar{\omega}^{\bar{l}}+\mathcal{O}\left(\omega^{3}\right) .
$$

Therefore as long as the sectional curvature is positive definite we can make a metastable state by adding the superpotential $W(X)=k_{i} \omega^{i}$.

It would be useful to find a sufficient condition for the positivity. In general, the curvature of the Kähler manifold is

$$
R_{i k \bar{l}}^{s}=g^{s \bar{j}} g^{m \bar{n}} \bar{\partial}_{\bar{j}} g_{m \bar{l}} \bar{\partial}_{\bar{n}} g_{i \bar{k}}-g^{s \bar{j}} \bar{\partial}_{\bar{j}} \partial_{i} g_{k \bar{l}} .
$$

Therefore when the second term at a point $X_{0}$ is vanishing, the potential becomes

$$
V=g^{k \bar{l}} k_{k} \bar{k}_{\bar{l}}+g^{k \bar{l}}\left(k_{i} \partial_{i} g_{s \bar{l}} \omega^{s}\right)\left(\bar{k}_{j} \bar{\partial}_{\bar{j}} g_{k \bar{m}} \bar{\omega}^{\bar{m}}\right)+\mathcal{O}\left(\omega^{3}\right) \text {. }
$$

Thus, we obtain a sufficient condition for the stability

$$
\left.\partial_{i} \bar{\partial}_{\bar{j}} g_{k \bar{l}}\right|_{X_{0}}=\left.0 \quad \& \quad k_{i} \partial_{i} g_{j \bar{k}}\right|_{X_{0}} \neq 0 \quad \text { sufficient condition } .
$$

When the first equation holds everywhere (except singular points) in the $X$ space, then the metric can be written in terms of a holomorphic function. Equivalently the kinetic term can be written as

$$
\operatorname{Im} \int d^{4} \theta X^{i \dagger} \frac{\partial \mathcal{F}(X)}{\partial X^{i}}
$$


This is the case of Seiberg-Witten theories [49]. As was shown in [17], in this case the Kähler normal coordinate can be written in terms of the $\mathcal{F}(X)$,

$$
\omega^{i}=X^{i}+m^{i j} \frac{\partial \mathcal{F}}{\partial X_{j}}
$$

where $m^{i j}$ satisfies $1+\bar{m}^{i j} \partial_{i} \partial_{j} \mathcal{F}\left(X_{0}\right)=0$. The vacuum obtained in this way breaks all supersymmetry unless the theory have an extended supersymmetry [17].

\section{References}

[1] E. Witten, "Dynamical Breaking Of Supersymmetry," Nucl. Phys. B 188, 513 (1981).

[2] I. Affleck, M. Dine and N. Seiberg, "Calculable Nonperturbative Supersymmetry Breaking," Phys. Rev. Lett. 52, 1677 (1984).

[3] I. Affleck, M. Dine and N. Seiberg, "Dynamical Supersymmetry Breaking In FourDimensions And Its Phenomenological Implications," Nucl. Phys. B 256, 557 (1985).

[4] M. Dine, W. Fischler and M. Srednicki, "Supersymmetric Technicolor," Nucl. Phys. B 189, 575 (1981).

[5] S. Dimopoulos and S. Raby, "Supercolor," Nucl. Phys. B 192, 353 (1981).

[6] M. Dine and W. Fischler, "A Phenomenological Model Of Particle Physics Based On Supersymmetry," Phys. Lett. B 110, 227 (1982).

[7] L. Alvarez-Gaume, M. Claudson and M. B. Wise, "Low-Energy Supersymmetry," Nucl. Phys. B 207, 96 (1982).

[8] M. Dine, A. E. Nelson and Y. Shirman, "Low-Energy Dynamical Supersymmetry Breaking Simplified," Phys. Rev. D 51, 1362 (1995) [arXiv:hep-ph/9408384].

[9] M. Dine, A. E. Nelson, Y. Nir and Y. Shirman, "New tools for low-energy dynamical supersymmetry breaking," Phys. Rev. D 53, 2658 (1996) [arXiv:hep-ph/9507378].

[10] G. F. Giudice and R. Rattazzi, "Theories with gauge-mediated supersymmetry breaking," Phys. Rept. 322, 419 (1999) [arXiv:hep-ph/9801271]. 
[11] M. A. Luty, "2004 TASI lectures on supersymmetry breaking," arXiv:hepth/0509029.

[12] K. Intriligator, N. Seiberg and D. Shih, "Dynamical SUSY breaking in meta-stable vacua," JHEP 0604, 021 (2006) [arXiv:hep-th/0602239].

[13] K. I. Izawa and T. Yanagida, Prog. Theor. Phys. 95, 829 (1996) [arXiv:hepth/9602180];

K. A. Intriligator and S. D. Thomas, Nucl. Phys. B 473, 121 (1996) [arXiv:hepth/9603158].

[14] K. A. Intriligator and N. Seiberg, "Lectures on supersymmetric gauge theories and electric-magnetic duality," Nucl. Phys. Proc. Suppl. 45BC, 1 (1996) [arXiv:hepth/9509066].

[15] K. Intriligator and N. Seiberg, "Lectures on supersymmetry breaking," arXiv:hep$\mathrm{ph} / 0702069$.

[16] H. Ooguri, Y. Ookouchi and C. S. Park, "Metastable Vacua in Perturbed SeibergWitten Theories," arXiv:0704.3613 [hep-th].

[17] J. Marsano, H. Ooguri, Y. Ookouchi and C. S. Park, "Metastable Vacua in Perturbed Seiberg-Witten Theories, Part 2: Fayet-Iliopoulos Terms and Kähler Normal Coordinates," Nucl. Phys. B 798, 17 (2008) [arXiv:0712.3305 [hep-th]].

[18] G. Pastras, "Non supersymmetric metastable vacua in $\mathrm{N}=2$ SYM softly broken to $\mathrm{N}=1, "$ arXiv:0705.0505 [hep-th].

[19] H. Ooguri and Y. Ookouchi, "Meta-stable supersymmetry breaking vacua on intersecting branes," Phys. Lett. B 641, 323 (2006) [arXiv:hep-th/0607183].

[20] S. Franco, I. Garcia-Etxebarria and A. M. Uranga, "Non-supersymmetric metastable vacua from brane configurations," JHEP 0701, 085 (2007) [arXiv:hepth/0607218].

[21] I. Bena, E. Gorbatov, S. Hellerman, N. Seiberg and D. Shih, "A note on (meta)stable brane configurations in MQCD," JHEP 0611, 088 (2006) [arXiv:hepth/0608157]. 
[22] H. Ooguri and Y. Ookouchi, "Landscape of supersymmetry breaking vacua in geometrically realized gauge theories," Nucl. Phys. B 755, 239 (2006) [arXiv:hepth/0606061].

[23] R. Argurio, M. Bertolini, S. Franco and S. Kachru, "Metastable vacua and Dbranes at the conifold," JHEP 0706, 017 (2007) [arXiv:hep-th/0703236].

[24] O. Aharony, S. Kachru and E. Silverstein, "Simple Stringy Dynamical SUSY Breaking," arXiv:0708.0493 [hep-th].

[25] Y. Nakayama, M. Yamazaki and T. T. Yanagida, "Moduli Stabilization in Stringy ISS Models," arXiv:0710.0001 [hep-th].

[26] M. Buican, D. Malyshev and H. Verlinde, "On the Geometry of Metastable Supersymmetry Breaking," arXiv:0710.5519 [hep-th].

[27] R. Kitano, "Dynamical GUT breaking and mu-term driven supersymmetry breaking," Phys. Rev. D 74, 115002 (2006) [arXiv:hep-ph/0606129].

[28] R. Kitano, "Gravitational gauge mediation," Phys. Lett. B 641, 203 (2006) [arXiv:hep-ph/0607090].

[29] M. Dine and J. D. Mason, "Dynamical Supersymmetry Breaking and Low Energy Gauge Mediation," arXiv:0712.1355 [hep-ph].

[30] R. Kitano, H. Ooguri and Y. Ookouchi, "Direct mediation of meta-stable supersymmetry breaking," Phys. Rev. D 75, 045022 (2007) [arXiv:hep-ph/0612139].

[31] C. Csaki, Y. Shirman and J. Terning, "A simple model of low-scale direct gauge mediation," JHEP 0705, 099 (2007) [arXiv:hep-ph/0612241].

[32] H. Murayama and Y. Nomura, "Gauge mediation simplified," Phys. Rev. Lett. 98, 151803 (2007) [arXiv:hep-ph/0612186].

[33] H. Murayama and Y. Nomura, "Simple scheme for gauge mediation," Phys. Rev. D 75, 095011 (2007) [arXiv:hep-ph/0701231].

[34] A. Amariti, L. Girardello and A. Mariotti, "On meta-stable SQCD with adjoint matter and gauge mediation," Fortsch. Phys. 55, 627 (2007) [arXiv:hepth/0701121]. 
[35] C. Cheung, A. L. Fitzpatrick and D. Shih, "(Extra)Ordinary Gauge Mediation," JHEP 0807, 054 (2008) [arXiv:0710.3585 [hep-ph]].

[36] M. Ibe and R. Kitano, "Sweet Spot Supersymmetry," JHEP 0708, 016 (2007) [arXiv:0705.3686 [hep-ph]].

[37] M. Ibe and R. Kitano, "Minimal Direct Gauge Mediation," Phys. Rev. D 77, 075003 (2008) [arXiv:0711.0416 [hep-ph]].

[38] S. A. Abel, C. Durnford, J. Jaeckel and V. V. Khoze, "Patterns of Gauge Mediation in Metastable SUSY Breaking," JHEP 0802, 074 (2008) [arXiv:0712.1812 [hep-ph]].

[39] N. Haba and N. Maru, "A Simple Model of Direct Gauge Mediation of Metastable Supersymmetry Breaking," Phys. Rev. D 76, 115019 (2007) [arXiv:0709.2945 [hep$\mathrm{ph}]$.

[40] S. Ray, "Some properties of meta-stable supersymmetry-breaking vacua in WessZumino models," Phys. Lett. B 642, 137 (2006) [arXiv:hep-th/0607172].

[41] D. Shih, "Spontaneous R-symmetry breaking in O'Raifeartaigh models," JHEP 0802, 091 (2008) [arXiv:hep-th/0703196].

[42] K. A. Intriligator, N. Seiberg and D. Shih, "Supersymmetry Breaking, RSymmetry Breaking and Metastable Vacua," JHEP 0707, 017 (2007) [arXiv:hepth/0703281].

[43] L. Ferretti, "R-symmetry breaking, runaway directions and global symmetries in O'Raifeartaigh models," JHEP 0712, 064 (2007) [arXiv:0705.1959 [hep-th]].

[44] H. Abe, T. Kobayashi and Y. Omura, "R-symmetry, supersymmetry breaking and metastable vacua in global and local supersymmetric theories," JHEP 0711, 044 (2007) [arXiv:0708.3148 [hep-th]].

[45] D. Marques and F. A. Schaposnik, "Explicit R-Symmetry Breaking and Metastable Vacua," arXiv:0809.4618 [hep-th].

[46] J. Polonyi, "Generalization Of The Massive Scalar Multiplet Coupling To The Supergravity," Hungary Central Inst Res - KFKI-77-93 (77,REC.JUL 78) $5 p$. 
[47] L. Alvarez-Gaume, D. Z. Freedman and S. Mukhi, "The Background Field Method And The Ultraviolet Structure Of The Supersymmetric Nonlinear Sigma Model," Annals Phys. 134, 85 (1981). C. M. Hull, A. Karlhede, U. Lindstrom and M. Rocek, "Nonlinear Sigma Models And Their Gauging In And Out Of Superspace," Nucl. Phys. B 266, 1 (1986).

[48] K. Higashijima and M. Nitta, "Kähler normal coordinate expansion in supersymmetric theories," Prog. Theor. Phys. 105, 243 (2001) [arXiv:hep-th/0006027].

[49] N. Seiberg and E. Witten, "Electric - magnetic duality, monopole condensation, and confinement in $\mathrm{N}=2$ supersymmetric Yang-Mills theory," Nucl. Phys. B 426, 19 (1994) [Erratum-ibid. B 430, 485 (1994)] [arXiv:hep-th/9407087].

[50] G. F. Giudice and R. Rattazzi, "Extracting Supersymmetry-Breaking Effects from Wave-Function Renormalization," Nucl. Phys. B 511, 25 (1998) [arXiv:hep$\mathrm{ph} / 9706540]$.

[51] N. Arkani-Hamed, G. F. Giudice, M. A. Luty and R. Rattazzi, "Supersymmetrybreaking loops from analytic continuation into superspace," Phys. Rev. D 58, 115005 (1998) [arXiv:hep-ph/9803290].

[52] E. Witten, "Mass Hierarchies In Supersymmetric Theories," Phys. Lett. B 105, 267 (1981).

[53] A. Giveon, A. Katz, Z. Komargodski and D. Shih, "Dynamical SUSY and Rsymmetry breaking in SQCD with massive and massless flavors," JHEP 0810, 092 (2008) [arXiv:0808.2901 [hep-th]].

[54] K. Intriligator, D. Shih and M. Sudano, "Surveying Pseudomoduli: the Good, the Bad and the Incalculable," arXiv:0809.3981 [hep-th].

[55] S. P. Martin and M. T. Vaughn, "Two Loop Renormalization Group Equations For Soft Supersymmetry Breaking Couplings," Phys. Rev. D 50, 2282 (1994) [Erratumibid. D 78, 039903 (2008)] [arXiv:hep-ph/9311340]. 